\title{
Oxidation State Assignment in Cerium Nanoclusters: Conflicting Conclusions from Single Crystal X-ray Diffraction and Spectroscopic Data
}

\author{
Jeffery A. Bertke, ${ }^{a}$ Jennifer N. Wacker, ${ }^{b}$ and Karah E. Knope ${ }^{\mathrm{c}}$ \\ ${ }^{\text {a }}$ Georgetown University, Department of Chemistry, $37^{\text {th }}$ and O Sts. NW, Washington, DC 20057, \\ USA,jb2667@georgetown.edu \\ ${ }^{\mathrm{b}}$ Georgetown University, Department of Chemistry, 37 ${ }^{\text {th }}$ and O Sts. NW, Washington, DC 20057, \\ USA, jnw37@georgetown.edu \\ ${ }^{\mathrm{c}}$ Georgetown University, Department of Chemistry, $37^{\text {th }}$ and O Sts. NW, Washington, DC 20057, \\ USA, kek44@georgetown.edu
}

Over the last decade, ceria nanoparticles have received much attention as an alternative to bulk cerium dioxide $\left(\mathrm{CeO}_{2}\right)$ due to their increased reactivity at lower temperatures. Further interest in Ce based clusters and nanoparticles stems from the nuclear community as cerium has historically been used as a surrogate for plutonium owing to their similar ionic radii, similar coordination environments, and accessible 3+/4+ redox couples. Several cerium clusters have been isolated using organic capping ligands to passivate the surface, thereby halting further oligomeric growth. Clusters capped by inorganic ligands, such as $\mathrm{Cl}^{-}$, are a bit more elusive. Yet our group has recently harnessed nonbonding interactions to isolate several Ce nanoclusters capped by chloride ions and water molecules; however, the valence state assignment of the Ce sites and the $\mu_{3}-\mathrm{O} / \mathrm{OH}$ sites on the cluster surface remains unclear. In one such example, we have crystallized a compound whose structure is built from $\mathrm{Ce}_{38}$ clusters. Analysis by single crystal X-ray diffraction and bond valence summation point towards exclusively tetravalent cerium but there is ambiguity in the assignment of $\mu_{3}-\mathrm{O} / \mathrm{OH}$ sites and the occupancy of outer sphere cations that charge balance the clusters, both of which have implications on the Ce oxidation state assignment. Additionally, other experimental data including X-ray absorption and X-ray photoelectron spectroscopies suggest the presence of $\mathrm{Ce}^{3+}$ in the cluster; charge compensation of the $\mathrm{Ce}^{4+} / \mathrm{Ce}^{3+}$ ratio can be accounted for through the relative ratio of $\mu_{3}-\mathrm{O} / \mathrm{OH}$ surface sites. Several plausible formula for the clusters have been determined. Taking into account both the SCXRD and spectroscopic data, "would you publish this"? 